\title{
2012 CSHP National Awards Program Winners Programme national des prix 2012 de la SCPH : lauréats et lauréates
}

The winner of the Distinguished Service Award (Johnson \& Johnson Family of Companies) is Carolyn Bornstein (Newmarket, ON).

The winner of the Isabel E. Stauffer Meritorious Service Award (sponsored by Pharmaceutical Partners of Canada Inc.) is Judy Chong (Mississauga, ON).
The winners of the New Hospital Pharmacy Practitioner Award (sponsored by Sandoz Canada Inc.) are Christina Adams (Pembroke, ON) and Erin Marie Yakiwchuk (Saskatoon, SK).

The winner of the Hospital Pharmacy Student Award (sponsored by the Canadian Society of Hospital Pharmacists [CSHP] and the Canadian Association of Pharmacy Students and Interns [CAPSI]) is Sarah Hasenbank (Edmonton, AB).
Management and Leadership Best Practices Award Sponsored by Apotex Inc.

1. Organizational Restructuring of Regional Pharmacy Services to Facilitate Pharmacy Practice Model Change (completed at Winnipeg Regional Health Authority, Winnipeg, MB) Kevin W Hall, Colette B Raymond, Donna M M Woloschuk, and Nicholas Honcharik

Sponsored by Hospira Healthcare Corporation

2. A Survey of Institutional Pharmacists' Involvement in and Attitudes towards Research (completed at the University of Alberta, Edmonton, AB)

Joseph E Blais, Sheila L Walter, Dean T Eurich, Ross T Tsuyuki, and Sheri L Koshman

Patient Care Enhancement Award

Sponsored by AstraZeneca Canada Inc.

1. Evaluating the Role of Pharmacist Teachers in Canadian Family Medicine Residency Programs: A Qualitative Analysis (completed at West Winds Primary Health Centre, Saskatoon, SK)

Derek Jorgenson and Anne Marie Whelan

Sponsored by TEVA Canada Ltd.

2. Development and Implementation of a Pediatric Emergency Department (ED) Asthma Action Plan and Prescription (AAPP) (completed at Children's Hospital of Eastern Ontario, Ottawa, ON)

Danica Irwin and Régis Vaillancourt

Pharmacotherapy Best Practices Award

Sponsored by Merck Canada Inc.

1. A Pharmacist-Directed Toxicity Management Program for Patients Receiving Capecitabine: Implementation and Evaluation (completed at the Horizon Health Network The Moncton Hospital, Moncton, NB)

Melissa Hawkins, Michael LeBlanc, and Gisia Pisegna

Sponsored by Pfizer Canada Inc.

2. Evaluation of a Prospective Audit and Feedback Program in Critical Care: A Controlled Interrupted Time Series Analysis (completed at Sunnybrook Health Sciences Centre, Toronto, ON)

Marion Elligsen and Sandra A N Walker
Safe Medication Practices Award Sponsored by Baxter Corporation

1. Extending Hospital-Based Medication Incident Reporting to Enhance Medication Safety and Continuous Quality Assurance in Pharmacy Practice (completed at the Institute for Safe Medication Practices Canada, Toronto, ON) Certina Ho

Sponsored by Hospira Healthcare Corporation

2. Implementation and Evaluation of a Medication Reconciliation Tool on Internal Hospital Transfer (completed at Horizon Health Network - The Moncton Hospital, Moncton, NB) Natalie LeBlanc, Timothy MacLaggan, Leslie Manuel, Julie Levesque, and Rochelle Johnston

Specialties in Pharmacy Practice Award

Sponsored by Pharmascience Inc.

1. Oseltamivir Pharmacokinetics in Morbid Obesity (OPTIMO

Trial) (completed at IWK Health Sciences Centre, Halifax, NS)

Lucas M Thorne-Humphrey and Kathy L Slayter

Sponsored by Hospira Healthcare Corporation

2. Interactions between Antiretroviral Agents and Chemotherapy Regimens for the Treatment of Lymphoma: A Quick Reference

Guide (completed at University Health Network, Toronto, ON) Alison YJ Wong, Alice Tseng, Jack Seki, and Pam Ng

Teaching, Learning, and Education Award Sponsored by Eli Lilly Canada Inc.

1. An Online e-Learning Patient Education Tool for Solid Organ Transplant Recipients (completed at Toronto General Hospital, Toronto, ON)

Jennifer Harrison

The award-winning abstracts are published exactly as submitted by the authors and have not undergone any copyediting by the Canadian Journal of Hospital Pharmacy.

Le Journal canadien de la pharmacie hospitalière n'a pas soumis les résumés gagnant des prix à une révision linguistique et les publie ici tels que remis par les auteurs. 


\section{Organizational Restructuring of Regional Pharmacy Services to Facilitate Pharmacy Practice Model Change}

Management and Leadership Best Practices Award, sponsored by Apotex Inc.

Kevin W Hall', Colette B Raymond', Donna M M Woloschuk²,

Nicholas Honcharik

${ }^{1}$ Faculty of Pharmacy, University of Alberta, Edmonton, $A B$

${ }^{2}$ Winnipeg Regional Health Authority, Winnipeg, $M B$

Background: Prior to restructuring, the Winnipeg Regional Health Authority's Pharmacy Program (WRHAPP) coordinated the delivery of pharmacy services at eight facilities with different drug distribution systems and pharmacy practice models. Pharmacy Managers had matrix accountability to their site and the WRHAPP.

Objectives: A three year pharmacy change initiative was approved, with the objectives of: i) creating a single line of staff accountability to the WRHAPP, ii) expanding, and standardizing the use of information/automation technologies, iii) focusing pharmacists on direct patient care activities, iv) expanding the role of pharmacy technicians to encompass responsibility for most drug distribution activities, v) creating management positions for pharmacy technicians, and vi) developing regional teams of specialized pharmacists, aligned with the WRHA's clinical program model.

Methods: Changes in the roles of pharmacists, technicians, and automation technology were facilitated by: i) the use of a structured project management approach, ii) the development and delivery of targeted educational/training programs, and iii) a consistent, repetitive communication strategy

Conclusions: The new model optimizes the use of pharmacy staff and automation technologies and positions the WRHAPP to efficiently deliver high quality, patient-focused pharmacy services.

Key words: Pharmacy Practice Model, Automation, Project Management

\section{A Survey of Institutional Pharmacists' Involvement} in and Attitudes towards Research

\section{Management and Leadership Best Practices Award, sponsored by Hospira Healthcare Corporation}

Joseph E Blais', Sheila L Walter', Dean T Eurich ${ }^{2}$, Ross T Tsuyuki, Sheri L Koshman

${ }^{1}$ Pharmacy Services, Alberta Health Services, Edmonton, $A B$

${ }^{2}$ School of Public Health, University of Alberta, Edmonton, $A B$

${ }^{3}$ Division of Cardiology, University of Alberta, Edmonton, $A B$

Background: Research is critical for the future of pharmacy yet institutional pharmacists' current research activities and perceptions towards research are largely unknown.

Objectives: To describe institutional pharmacists' involvement in research activities and to assess the attitudes of pharmacists towards research.

Methods: We conducted a cross-sectional survey of all institutional pharmacists within Alberta Health Services, Alberta, Canada, in March 2011. The survey explored involvement in research, current research activities, and attitudes towards research.

Results: Overall response rate was 45\% (286/636). Respondents were predominantly female $(80 \%)$, practiced in urban institutions $(76 \%)$ and reported a primary clinical role (61\%). Over half of pharmacists indicated having involvement in research (168/286 [60\%]) with 43\% of pharmacists $(119 / 280)$ currently participating in research activities but only 26\% (72/280) leading research projects. Pharmacists tended to agree $(94 \%)$ that research was important to the profession of pharmacy. Almost all respondents (99\%) felt that pharmacists should be involved in research, but indicated additional time and training were barriers to their personal involvement.

Conclusion: While the majority of institutional pharmacists in Alberta have had involvement in research projects, few were involved in leading research. There is strong support from pharmacists to be involved in research.

Key words: research, institutional pharmacist, attitudes, involvement, activities

\section{Evaluating the Role of Pharmacist Teachers in Canadian Family Medicine Residency Programs: A Qualitative Analysis \\ Patient Care Enhancement Award, sponsored by AstraZeneca Canada Inc.}

Derek Jorgenson', Andries Muller', Anne Marie Whelan'

${ }^{1}$ University of Saskatchewan, Saskatoon, SK

${ }^{2}$ Dalhousie University, Halifax, NS

Background: A 2009 survey found >25\% of Canadian family medicine residency programs have a pharmacist teaching residents; however, little is known about the impact of these pharmacists.

Objective: Evaluate the role of pharmacist teachers within Canadian family medicine residency programs.

Methods: One-on-one interviews with residents, physicians and pharmacists from the 40 Canadian family medicine residency programs that employ a pharmacist teacher. Interviews continued until data saturation was observed. Transcripts were analyzed using grounded theory analysis (inductive and deductive) to identify themes. Codes were entered into NVivo software. Themes were sent to participants to confirm validity.

Results: 11 residents, 6 physicians and 17 pharmacists participated. Four themes emerged from deductive analyses regarding the impact of pharmacists on residents: (1) Improved drug knowledge (2) Improved confidence (3) Improved patient care (4) Appreciation for pharmacist expertise. Five additional themes emerged from inductive analyses: (1) Desire to expand pharmacist teaching (2) Impact on collaboration (3) Impact on faculty (4) Lack of criticism of the role (5) No formal curriculum utilized.

Conclusions: The role of pharmacist teachers is valued in Canadian family medicine residency programs and consideration could be made to expand the role. Developing a formal curriculum is recommended. Key words: family medicine residency, pharmacist teacher, qualitative

\section{Development and Implementation of a Pediatric Emergency Department (ED) Asthma Action Plan and Prescription (AAPP)}

\section{Patient Care Enhancement Award, sponsored by TEVA Canada Ltd.}

Danica Irwin, Régis Vaillancourt, Roger Zemek, Elena Pascuet Children's Hospital of Eastern Ontario, Ottawa, ON

Background: National and international asthma guidelines endorse asthma action plan (AP) use. Preprinted prescription incorporation into the AP should enhance physician use. Provision of information in visual and text format addresses varied health literacy levels.

Objectives: To develop and implement a pediatric AP combined with a prescription based on validated pictograms accessible to the patient, pharmacist and physician. 
Methods: The Asthma Action Plan and Prescription (AAPP) was developed by a working group from ED, Respirology and Pharmacy, with additional input from the Ontario College of Pharmacists and the Institute for Safe Medication Practices. Evidence-based content was enhanced with pictograms of asthma control/triggers, with prescriptions embedded. Education was sent to ED staff via emails, posters and educational sessions. The ED pharmacist, via the region's pharmacy email system/website and telephone, instructed retail pharmacies how to incorporate the AAPP into practice.

Results: Since implementation in March 2011, more than 750 AAPPs have been used. Response is positive based on physician, pharmacist and patient feedback. An analysis of effectiveness is planned.

Conclusions: An AAPP has been developed and implemented to enhance patient, pharmacist and physician asthma management. The AAPP facilitates physician use of the document and subsequent enhanced rational medication prescribing.

Key words: asthma action plan, emergency department, health literacy

\section{A Pharmacist-Directed Toxicity Management Program for Patients Receiving Capecitabine: Implementation and Evaluation}

\section{Pharmacotherapy Best Practices Award, sponsored} by Merck Canada Inc.

Melissa Hawkins, Michael LeBlanc, Gisia Pisegna

Horizon Health Network, The Moncton Hospital, Moncton, NB

Background: Capecitabine is an oral pro-drug of the cytotoxic agent 5-fluorouracil used in the treatment of several types of cancer. As more oral chemotherapy agents come to market, there is interest in how pharmacists in oncology can adapt their practices to best provide patient care.

Objective: To establish and evaluate the impact of a pharmacist-led toxicity management program for patients being treated with capecitabine.

Methods: Implementation and prospective evaluation of the program was conducted using a matched historical cohort. Pharmacists performed weekly toxicity assessments. Interventions were made using developed algorithms and evidence-informed guidelines. Patient quality of life and satisfaction with the program were also evaluated.

Results: Seventeen patients were enrolled with 17 retrospective matches subsequently identified. There was a trend towards a decreased requirement for dose modifications and a significant decrease in the number of days of treatment delay in the intervention group (94 vs. 14 days, $\mathrm{p}=0.03$ ). The mean number of toxicities identified per patient was 5.3 in the prospective group compared to 1.6 in the retrospective group $(\mathrm{p}<0.001)$.

Conclusions: This toxicity management program represented an expanded role for clinical oncology pharmacists. It was well received by patients and considered safe and effective.

Key words: capecitabine, oncology pharmacy, clinical pharmacy services, chemotherapy toxicity.

\section{Evaluation of a Prospective Audit and Feedback Program in Critical Care: A Controlled Interrupted Time Series Analysis}

\section{Pharmacotherapy Best Practices Award, sponsored by} Pfizer Canada Inc.

Marion Elligsen ${ }^{I}$,Sandra A N Walker ${ }^{1,2,3}$, Ruxandra Pinto ${ }^{4}$, Andrew Simor ${ }^{3,5}$, Samira Mubareka ${ }^{3,4,5}$, Anita Rachlis ${ }^{3,5}$, Vanessa Allen ${ }^{3,5,6}$, Nick Daneman ${ }^{3,4,5,7}$
${ }^{1}$ Department of Pharmacy, Sunnybrook Health Sciences Centre, Toronto, ON ${ }^{2}$ Leslie L. Dan Faculty of Pharmacy, University of Toronto, Toronto, ON ${ }^{3}$ Department of Microbiology and Division of Infectious Diseases, Sunnybrook Health Sciences Centre, Toronto, ON

${ }^{4}$ Sunnybrook Research Institute, Toronto, ON

${ }^{5}$ Faculty of Medicine, University of Toronto, Toronto, $O N$

${ }^{6}$ Public Health Ontario, Toronto, ON

${ }^{7}$ Institute for Clinical Evaluative Sciences, Toronto, $O N$

Rationale: While there is support for antibiotic stewardship programs in critical care, most data comes from uncontrolled before-and-after studies that are prone to bias and temporal confounding.

Objective: We aimed to rigorously evaluate the impact of a prospective audit-and-feedback program in critical care patients, using a controlled, interrupted time series analysis.

Methods: The antimicrobial stewardship team provided prospective audit-and-feedback for all patients in level III ICUs on their 3rd or 10th day of targeted broad-spectrum antibiotic therapy. Antibiotic use during the one year pilot was compared to the year prior using a controlled interrupted time series analysis. Secondary outcomes included overall antibiotic use, gram negative bacterial susceptibility, rates of nosocomial C.difficile infection, length of stay and mortality.

Results: Broad-spectrum antibiotic use significantly decreased (119 DOTs/1000 PDs; SE=37.9; $\mathrm{p}=0.0054$ ) with the implementation of the program, with no change in either control wards or use of control medications (stress ulcer prophylaxis). The incidence of $C$. difficile infections decreased from 11 to 6 cases, while increasing in the control wards from 87 to 116 cases $(p=0.04)$. Overall gram negative susceptibility to meropenem increased. Length of stay and mortality did not change.

Conclusions: Through collaboration with critical care, a successful prospective audit-and-feedback program was implemented in the level III ICUs.

Key words: Antimicrobial Stewardship, Critical Care, Intensive Care

\section{Extending Hospital-Based Medication Incident Reporting to Enhance Medication Safety and Continuous Quality Assurance in Pharmacy Practice Safe Medication Practices Award, sponsored by Baxter Corporation}

Certina Ho ${ }^{1,2}$, Roger Cheng', Calvin Poon ${ }^{1,2}$, Patricia Hun ${ }^{1,2}$, Gary Lee ${ }^{l}$, Joe O'Leary', Kristian Duwyn ${ }^{l}$, Medina Kadija ${ }^{l}$, Carol Lee ${ }^{l}$, Sanaz Riahi ${ }^{1,2}$ ${ }^{1}$ Institute for Safe Medication Practices Canada, Toronto, ON ${ }^{2}$ School of Pharmacy, University of Waterloo, Kitchener, ON

Background: Medication safety / risk management is a relatively foreign terminology in community pharmacy practice when compared to other health care settings in Canada. This stems from the lack of a medication incident reporting tool tailored for community pharmacies, thereby discouraging community pharmacies from learning past mistakes.

Objective: Based on learning and experience acquired from hospitalbased incident reporting, the ISMP Canada Community Pharmacy Incident Reporting (CPhIR) program was created specifically for community / ambulatory pharmacies.

Methods: After multiple iterations of feedback, pilot-testing, and consultation with community pharmacy practitioners, CPhIR is now available to community / ambulatory pharmacies. Reported incidents are analyzed anonymously using a qualitative aggregate analysis approach with key findings disseminated back to frontline users by ISMP Canada. 


\section{CSHP NATIONAL AWARDS PROGRAM / PROGRAMME NATIONAL DES PRIX 2012 DE LA SCPH}

Results: As of August 2011, there are over 300 registered CPhIR users. An accumulative total of 17,000 incidents have been anonymously reported. CPhIR provides users with a secure online interface to document medication incidents, export data for analysis, and view comparisons of individual pharmacy and aggregate data.

Conclusions: The innovative CPhIR program provides the necessary tools to empower community pharmacies to enhance safe medication practices. Through anonymous reporting, community pharmacies can analyze medication incidents, identify root causes, and consequently implement system-based strategies for continuous quality assurance.

Key words: Community Pharmacy, Ambulatory Pharmacy, Incident Reporting, Medication Incidents, Near Miss, Continuous Quality Assurance

\section{Implementation and Evaluation of a Medication Reconciliation Tool on Internal Hospital Transfer Safe Medication Practices Award, sponsored by Hospira Healthcare Corporation \\ Natalie LeBlanc, Timothy MacLaggan, Leslie Manuel, Julie Levesque, Rochelle Johnston, Julie Weir \\ Horizon Health Network, Zone 1 Moncton, Moncton, NB}

Background: Patients are susceptible to medication errors at the point of transfer, particularly in transfer from an intensive care unit to a general ward. Data to assess the optimal strategy in performing medication reconciliation at the time of transfer is currently limited.

Objectives: To determine if implementation of a medication reconciliation tool comprised of a patient-specific computer generated physician order form would result in a decrease in the number, type and severity of medication discrepancies that occur on internal hospital transfer.

Methods: Two groups of patients transferred from the intensive care unit to the surgery step-down unit were compared. Current practices with regard to transfer orders were provided to the control group and a patient-specific medication reconciliation tool (completed by the transferring physician) was utilized for the intervention group. Medication discrepancies at the time of transfer were identified by two independent reviewers.

Results: Fifty-two patients were included in the analysis. Mean number of discrepancies per-patient were significantly decreased in the intervention group compared to the control group $(0.96 \pm 1.5$ vs. 2.37 $\pm 1.8 ; \mathrm{p}=0.003)$. There was a significant reduction in the number of policy violation discrepancies $(0 \%$ vs. $45 \%$; $<<0.001)$ and in discrepancy severity $(\mathrm{p}<0.001)$ in the intervention group as compared to the control group.

Conclusion: Use of a patient-specific computer generated medication reconciliation tool to ensure that medications are reconciled at the time of transfer is expected to help improve patient safety.

Key words: medication reconciliation, internal hospital transfer, medication discrepancy, intensive care unit, patient safety

\section{Oseltamivir Pharmacokinetics in Morbid Obesity (OPTIMO Trial)}

Specialties in Pharmacy Practice Award, sponsored by Pharmascience Inc.

L M Thorne-Humphrey ${ }^{l, 2}, K B$ Goralskit, ${ }^{l, 3}, K$ L Slayter ${ }^{l, 2,4,5}$,

T F Hatchette ${ }^{2,4,6}, B$ L Johnston ${ }^{2,4}, S A M c N e i 2^{2,4,7,7}$, on behalf of the

OPTIMO Study Group
${ }^{\prime}$ College of Pharmacy, Dalhousie University, Halifax, NS

${ }^{2}$ Pharmacy Department, Capital District Health Authority Halifax

Infirmary, Halifax, NS

${ }^{3}$ Department of Pharmacology, Dalhousie University, Halifax, NS

${ }^{4}$ Department of Medicine, Dalhousie University, Halifax, NS

${ }^{5}$ Canadian Centre for Vaccinology, Halifax, NS

${ }^{6}$ Department of Pathology, Dalhousie University, Halifax, NS

'IWK Health Centre, Halifax, NS

Background: Detailed pharmacokinetics to guide oseltamivir (Tamiflu (B) dosing in morbidly obese patients is lacking.

Objectives: Characterize the single dose and steady state pharmacokinetics of oseltamivir and its active carboxylate metabolite in this population.

Methods: The OPTIMO trial was a single center non-randomized open label pharmacokinetic study of single dose and steady state oral oseltamivir phosphate and active metabolite in healthy morbidly obese and healthy, non-obese subjects.

Results: In the morbidly obese v.s. control subjects, respectively, the single-dose median oseltamivir: oral clearance, CL/F [840 v.s. 580 $\mathrm{L} \mathrm{h}^{-1}$ ] was higher; the area under the curve $\left(\mathrm{AUC}_{0-\infty}\right)$ [89 v.s. $132 \mathrm{ng} \mathrm{mL}$ ${ }^{1} \mathrm{~h}$ ] was lower and, the volume of distribution $\mathrm{V}_{\mathrm{d}} / \mathrm{F}[2320$ v.s. $1670 \mathrm{~L}$ ] was unchanged. In the morbidly obese v.s. control subjects, respectively, the single-dose median oseltamivir carboxylate CL/F, AUC $_{0-12 \mathrm{~h}}$ and $\mathrm{V}_{\mathrm{d}} / \mathrm{F}$ did not differ significantly. Similar results for oseltamivir and oseltamivir carboxylate $\mathrm{CL} / \mathrm{F}, \mathrm{AUC}_{0-12 \mathrm{~h}}$ and $\mathrm{V}_{\mathrm{d}} / \mathrm{F}$ values were observed in the multiple dose study.

Conclusions: Systemic exposure to oseltamivir is decreased but that of oseltamivir carboxylate is largely unchanged. Based on these pharmacokinetic data, an oseltamivir dose adjustment for body weight would not be needed in morbid obese individuals.

Key words: Oseltamivir, obesity, body mass index, obese, pharmacokinetics

\section{Interactions between Antiretroviral Agents and Chemotherapy Regimens for the Treatment of Lymphoma: A Quick Reference Guide}

Specialties in Pharmacy Practice Award, sponsored by Hospira Healthcare Corporation

Alison Y J Wong ${ }^{1,2,3}$, Alice Tseng ${ }^{1,2}$, Jack Seki ${ }^{1,2}$, Pam Ng ${ }^{l}$, Vishal Kukreti ${ }^{l}$

${ }^{1}$ University Health Network, Toronto, $\mathrm{ON}$

${ }^{2}$ University of Toronto, Toronto, $\mathrm{ON}$

${ }^{3} \mathrm{Mc}$ Gill University Health Centre, Montréal, QC

Background: Despite the success of antiretroviral therapy, HIV-infected individuals remain at higher risk of developing malignancies. Clinically important interactions between cART and chemotherapy agents have been described in the literature, but practical, comprehensive guidelines for clinical practice do not exist.

Objective: To provide a comprehensive summary of documented and potential interactions between antiretroviral agents (ARVs), common chemotherapy regimens used in the treatment of lymphoma, and supportive therapy.

Methods: A literature review was conducted through Ovid Medline 1948 - March 2011 using MeSH terms for individual antineoplastics, keywords for chemotherapy regimens, and available and emerging ARVs. Pertinent conference abstracts were identified through the International AIDS Society USA abstract search engine. Quality of evidence was evaluated according to an adapted GRADE system. 


\section{CSHP NATIONAL AWARDS PROGRAM / PROGRAMME NATIONAL DES PRIX 2012 DE LA SCPH}

Results: Thirteen documents were developed, summarizing potential interactions between $\mathrm{ARV}$ s and the following chemotherapy regimens: ABVD, BEACOPP, CHOP, CNS LYMPHOMA, CODOX-M, CVP, DHAP, ESHAP, GDP, ICE, IVAC, MINIBEAM, and supportive therapy. A summary of current principles of HIV treatment was also developed for non-HIV specialists.

Conclusion: This guide provides clinicians with a user-friendly tool which allows quick identification of possible ARV-chemotherapy interactions; clinical implications and practical management guidelines are included to facilitate optimal treatment of both disease states with minimal toxicity.

Key words: HIV infection, lymphoma, antiretrovirals, chemotherapy, interactions

\section{An Online e-Learning Patient Education Tool for Solid Organ Transplant Recipients \\ Teaching, Learning, and Education Award, sponsored by Eli Lilly Canada Inc.}

Jennifer Harrison ${ }^{l, 2}$, Julia Cervenko', Penny Demas-Clarke',

Bassem Hamandi, ${ }^{\prime, 2}$ Dipika Munyal', June Wang', Teresa Yi

${ }^{1}$ Department of Pharmacy, Toronto General Hospital, University Health

Network, Toronto, ON

${ }^{2}$ Leslie Dan Faculty of Pharmacy, University of Toronto, Toronto, ON

Background: CMedication regimens for solid organ transplant recipients (SOTR) are complex and non-adherence can lead to rejection and graft loss. Educational tools are needed to reinforce patient knowledge of transplant medications.

Objective: To develop a tool to support ongoing medication education needs of SOTR.
Methods: Program content was developed by pharmacists and reviewed by an interprofessional clinician group. Microsoft Power Point ${ }^{\circledR}$, Adobe $\AA$ Captivate $\AA$ and Dreamweaver ${ }^{\circledR}$ were used to create an e-learning internet-based format. A Virtual Patient Focus Group (VPFG) assessed usability and utility of the tool.

Results: The Transplant Medication Information Teaching Tool (TMITTC, www.TMITT.ca) launched in September 2010. Content is structured as medication courses and lessons, and includes interactive quizzes and medication summaries. 'Learning Prescriptions' direct patients to appropriate content to meet their learning objectives. The majority of VPFG members $(n=27)$ agreed or strongly agreed that the program was an effective way to learn, language and difficulty level were appropriate, and that they had the necessary computer skills to use the tool.

Conclusions: While further study is needed, use of an internet-based e-learning program to control content, sequence, pace and timing of education to achieve personal learning objectives appears to be an attractive and effective teaching modality in SOTR.

Key words: patient education, e-learning, internet, solid organ transplantation

\section{Advertisers' Index}

\begin{tabular}{lll}
\hline & Ad Page & Prescribing Information \\
\hline Hospira / Docetaxel & 4 & - \\
\hline Meda Valeant / Onsolis & 8,9 & $50-52$ \\
\hline Pharmaceutical Partners of Canada / Corporate & IFC & - \\
\hline Pharmaceutical Partners of Canada / Corporate & OBC & - \\
\hline Sandoz / Corporate & 2 & - \\
\hline Sandoz / Corporate & 7 & - \\
\hline
\end{tabular}

